\title{
RADIOCHEMICAL EXPERIMENTS FOR AN INTRODUCTORY COURSE IN PHYSICAL SCIENCE
}

\author{
H. C. GRIFFIN, M. G. CHEMALY \\ Department of Chemistry, University of Michigan, Ann Arbor MI 48109.1055 (USA) \\ (Received November 9, 1992) \\ A new curriculum in radiochemistry for undergraduate chemistry is described.
}

In response to trends in student interests and preparation, the Department of Chemistry of the University of Michigan has created a new curriculum for undergraduate chemistry. The overall structure of the "main sequence" and the courses in the first two years are described in a recent monograph. ${ }^{1}$ In brief, students with strong backgrounds in chemistry and other sciences begin with organic chemistry, modified to include significant emphasis on the principles of general chemistry in the context of organic chemistry. Alternatively, students take a one term course in general chemistry before continuing with the new normal sequence. Although either course can be chosen by students who do not have professional interests speciffically requiring chemistry, in practice relatively few of these students choose chemistry. For these students, or a very small fraction of them, we have designed several "theme" courses.

A major barier to creating new courses is the lack of a compatible textbook. The first of the one-term theme courses was based on a textbook. Seyhan EGE had already explored the idea of introducing organic chemistry earlier than usual and was drawn to Chemistry. A Search to Understand by HARRISON and WEAVER, for both its biochemical orientation and its emphasis on the reasoning of chemistry. She created a course designed to match the interest of students with some background in biology and chemistry.

We designed the second theme course. "The Atom", to draw from the more physical aspects of chemistry and from physics. When general chemistry in our curriculum was reduced to one term, nuclear chemistry was one of the topics which was dropped. In fact it had been given relatively little emphasis in recent years, even in the two term sequence of general chemistry. Students typically considered nuclear chemistry one of the most interesting parts of introductory chemistry, but many instructors were more confortable with other topics. And there never was time for everything.

We anticipated that a course not burdened by the need to cover prerequisites to a subsequent course could build on that student interest. Lecture topics in the course were (numbers refer to chapters in the textbook ${ }^{2}$ in the order in which they were used): 
1. The atomic nature of matter;

2. Chemical equations and reaction yields;

3. Chemical periodicity and the formation of simple compounds;

4. Types of chemical reactions;

5. The gaseous state;

16. Fundamental particles and nuclear chemistry;

17. Quantum mechanics and the hydrogen atom, and

18. Many-electron atoms and chemical bonding.

Both of the theme courses included laboratory. "The Atom" relied heavily on the Stephen Thompson's manual ${ }^{3}$ for small-scale laboratory work. When the students were ready to begin radiochemical work, they had investigated 8 of Thompson's 22 units, namely:

1. The system;

2. Spectroscopy; the interaction of light with matter;

3. Small-scale techniques and the absorption of light;

5. Instruments; what they do and what they don't;

7. Solutions and reactions;

15. Redox equilibria and electrochemistry;

18. Paper chromatography and liquid chromatography, and

19. Gas chromatography.

Our criteria for the radiochemical experiments were compatibility with the small-scale techniques introduced in these 8 units, relevance to periodic properties in the sense of groups, quantitative attention to errors (random statistics), and a general mutual reinforcement between lab and lecture (lecture not always coming first). We devised 4 nuclear chemistry units.

A. Solvent extraction and gamma-ray spectroscopy;

B. Paper chromatography and beta spectroscopy;

C. Neutron activation, and

D. Alpha spectroscopy.

For neutron activation we used a Pu-Be source, silver foils, and a Geiger-Mueller (GM) detector. We will say no more about this well-known experiment except to note that it emphasized induced activity, half life, and statistical uncertainty. The alpha-spectroscopy was intended to bring the laboratory work close to then current interests (levels of radon in university buildings), but the first three units took more time than anticipated and we dropped alpha spectroscopy from the schedule. Therefore this report is confined to the radiochemical experiments and the detectors used to follow the chemistry. 


\section{Materials and methods}

Our resources included a paraffin-moderated plutonium-beryllium source, a research reactor, a radiochemical laboratory, a variety of detectors (GM counters, $\mathrm{Nal}$ and $\mathrm{Ge}$ gamma-ray spectrometers, and plastic scintillators for beta-ray spectroscopy), and PC-based multichannel annalyzers. As we discuss later, much simpler equipment would suffice.

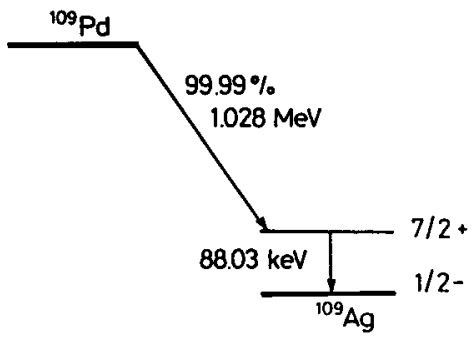

Fig. 1. Decay scheme of ${ }^{109} \mathrm{Pd}$

We chose 13.4-hour ${ }^{109} \mathrm{Pd}$ (see Fig. 1) as the main nuclide around which to construct the radiochemical units. Many factors went into the selection, but principal criteria were half life (long enough to transport, even interstate, and short enough to avoid long-term contamination if spilled) and radiation energies (gamma radiation easy to shield, beta radiation energetic enough to produce good spectra). Of course the chemistry of the chosen nuclide is important, but not so constraining. The ${ }^{109} \mathrm{Pd}$ was produced by irradiating a small amount (about $2 \mathrm{mg}$ ) of $\mathrm{Pd}$ metal in the reactor. We used two different samples of Pd metal, a rather pure metal and a another which contained significant amounts of impurities ("old Pd"). Each was dissolved in aqua regia and diluted to a few $\mathrm{ml}$. Generally the students worked with the impure material.

The solvent extraction and gamma-ray spectroscopy were carried out in two 3-hour lab periods. In the first period reactions with dimethylgloxime were explored, and gamma spectra of the activated Pd foils were obtained. For the second period a week later, additional foils were activated and the radiochemical work was carried out. Because arrangements with the radiation safety office had not been completed for teaching labs, work of the first two weeks was carried out in a radiochemical laboratory. Instructions to the students were as follows.

1. Reactions of $\mathrm{Ni}, \mathrm{Pd}$, and Au ions with dimethylglyoxime (DMG). Obtain $1 \mathrm{ml}$ each of $\mathrm{NiCl}_{2}, \mathrm{PdCl}_{2}$, and $\mathrm{AuCl}_{3}$ solutions. To the nickel solution add enough concentrated $\mathrm{NH}_{4} \mathrm{OH}$ to obtain a basic solution. Dilute to about $10 \mathrm{ml}$ with water. Add 1-2 $\mathrm{ml}$ of the 
dimethylglyoxime solution (standard solution for nickel determination). What do you observe? Is there a precipitate? What colors do you see?

Transfer the precipitate to a centrifuge tube and centrifuge it. Add some more DMG to the liquid left behind. Are there reactions?

Wash the precipitate with water and a drop of $\mathrm{NH}_{4} \mathrm{OH}$, let it dry, and describe it. Is it soluble in dilute $\mathrm{HCl}$ ?

To the Pd solution add enough dilute $\mathrm{HCl}$ to make the solution acid, and dilute to 10 ml. Add 1-2 ml of the DMG solution and observe the result. How is the precipitate different from that obtained with nickel?

Transfer and centrifuge. Test the residual liquid for Pd. Wash the precipitate with water and a drop of $\mathrm{HCl}$. Examine it. Is it soluble in $\mathrm{NH}_{4} \mathrm{OH}$ ? Could you use DMG to separate $\mathrm{Pd}$ from $\mathrm{Ni}$ ?

Make the gold solution basic with $\mathrm{NH}_{4} \mathrm{OH}$, dilute it to $10 \mathrm{ml}$, and add DMG. Is there a reaction?

Acidify with dilute $\mathrm{HCl}$, add a bit more DMG, and describe.

2. Extraction of Pd from a mixture of palladium and gold. Add $1 \mathrm{ml}$ of $\mathrm{Pd}$ solution to $1 \mathrm{ml}$ of Au solution in a test tube. Based on your observationss in part 1, devise a procedure to separate $\mathrm{Pd}$ from the solution. Try your procedure on half the $\mathrm{Pd}-\mathrm{Au}$ solution. (A review of the principles of solvent extraction was given here.) To the other half of your solution add enough dilute $\mathrm{HCl}$ to make it acidic. Add an equal volume of chloroform and 1-2 ml of DMG solution. Separate the chloroform layer (by small-scale techniques) and wash it with dilute nitric acid. Which layer contains the Pd?

Repeat the extraction with more chloroform and DMG, and combine the chloroform fractions. Add concentrated ammonia solution to the chloroform. Separate and retain the aqueous layer. The $\mathrm{Pd}$ ions can be reduced to $\mathrm{Pd}$ metal by addition of $\mathrm{Zn}$ powder.

3. Gamma-spectroscopy of activated $P d$. (This section was preceeded by a demonstration of the portable survey meter. Attention was given to background rates and readings for the Pd foils (see below) at various distances and through various absorbers.)

A sample of $\mathrm{Pd}$, about $2.5 \mathrm{mg}$, was placed in the reactor's core for 15 minutes. While in the core the sample was bombarded with neutrons at the rate of about $2 \cdot 10^{13} \mathrm{n} \cdot \mathrm{cm}^{-2} \cdot \mathrm{s}^{1}$ and a few atoms of ${ }^{108} \mathrm{Pd}$ were converted to ${ }^{109} \mathrm{Pd}$. Similar reactions may have occurred for any impurities present in the Pd. After your instructor has explained the use of the $\mathrm{Na}(\mathrm{TI})$ scintillation counter, obtain a gamma-spectrum of the sample and identify the main peaks and their energies. For ${ }^{109} \mathrm{Pd}$ we expect gamma-rays of about $88 \mathrm{keV}$ in energy. Activation of $\mathrm{Pt}$ would produce ${ }^{197} \mathrm{Pt}$, which emits gamma-rays of 77 and $191 \mathrm{keV}$. A neighboring element which can be seen by activation analysis even when present in very small quantities is $\mathrm{Au}$, which can be detected by the $412-\mathrm{keV}$ gamma-rays of ${ }^{198} \mathrm{Al}$. Based on your spectra, which of these radioactive 
species are present? Do you see any features in the spectrum not explained by these species?

4. Clearing up the ${ }^{109} \mathrm{Pd}$ source. Use the solvent extraction technique developed above to remove any impurities from the $\mathrm{Pd}$ sample. Be careful in handling the radioactive material. Wear your apron or lab coat, your safety glasses and gloves. Work in a tray with an absorbent pad in the bottom of the tray. Obtain gamma-ray spectra of each phase for each solvent extraction step. Relate the spectra to the chemical processes you expected.

5. Interaction of radiation with matter. Place the cleaned ${ }^{109} \mathrm{Pd}$ source about an inch from the Nal scintillation detector, obtain a gamma-ray spectrum, and record the count rate of the 88-keV peak. Repeat the process for several thicknesses of $\mathrm{Pb}$ sheet placed between the source and detector. Plot the count rate as a function of $\mathrm{Pb}$ thickness. Make a similar set of measurements with $\mathrm{Bi}$ absorbers.

A similar set of instructions was given for the laboratory unit involving paper chromatography and measurements with beta-rays. Tests were made $\mathrm{PdCl}_{2}, \mathrm{PtCl}_{2}$, and $\mathrm{AuCl}_{3}$ solutions spotted on paper. They were developed with ether-butanol-HCl $(1: 2: 1)$, $\mathrm{n}$ - butanol saturated with $10 \% \mathrm{HCl}$ and $10 \% \mathrm{HNO}_{3}$, and butanol-water- $\mathrm{HCl}$ $(7: 2: 1)$. (Ratios are by volume, and $\mathrm{HCl}$ and $\mathrm{HNO}_{3}$ refer to the concentrated acids.) Spots were detected by spraying the dried paper with a solution of $\mathrm{SnCl}_{2}$ and $\mathrm{Kl}$. Each student characterized one mobile by determining $R_{f}$ for each element. The results were combined (differences in results for a given property were resolved by discussing probable errors in measurement), and a best system was chosen. Each student used that system in an attempt to separate all three elements from one spot. Finally, the best system was used to separate the components in activated $\mathrm{Pd}$ solution. The dried paper was cut according to expected $R_{f}$ values, and the pieces were examined with beta- and gamma-ray spectrometers.

The paper chromatography work was done in a regular teaching laboratory. Students worked in trays as they had learned in Section 4. At the end of the laboratory period the trays were monitored with a portable GM detector, active absorbent pads were placed in plastic bags along with gloves the students had used, swipes were taken for subsequent counting (in case a question of contamination arose), and the trays were stacked in storage.

\section{Results}

The preliminary studies of solvent extraction properties (parts 1 and 2 above) went well in the sense of getting the right answers. However, interest lagged until the gamma-ray spectra were obtained. The spectra were easy to interpret. Both ${ }^{109} \mathrm{Pd}$ and 
${ }^{198} \mathrm{Au}$ were clearly present in the activated impure $\mathrm{Pd}$ foil. There was not enough ${ }^{197} \mathrm{Pt}$ to show in their spectra. The students were excited to see an impurity such as gold so clearly, but were disappointed not to see platinum which, by periodic properties, was expected to be a major contaminant. (We had explained that nickel does not show readily by activation analysis.) The students were slightly disappointed in getting only ten-fold improvement in the purity of Pd in one solvent extraction step.

Paper chromatography was judged to be much better than solvent extraction, and gamma-ray spectroscopy much more useful than beta spectroscopy. We had expected to discuss the beta spectra, which showed continuum shape and end-point rather clearly, in terms of the neutrino hypothesis, but they showed little interest in "understanding" something with so little use Similarly, they got good absorption curves for beta rays and gamma rays, but resisted discussing them. Finally, they accepted statistical uncertainty as a minor annoyance, but were unimpressed that the variations were approximately the square root of the number of counts.

\section{Discussion}

These results derive from the first offering of the course. Only 7 students participated in the nuclear chemistry units. During lab work there was almost constant discussion among the students and teaching assistant. In our design we assumed that discussion would take place, but it might not work as well with a more typical group of 20-25 students.

The stated purpose in the laboratory work was to make some sense (for the students) out of their observations and carry out chemical separations based on the chemical properties they had determined. The use of radioactive materials was intended to reinforce the analytical value of spectroscopy. These goals were certainly reached.

The minimum equipment needed for these experiments includes a GM survey meter and a NaI detector with multichannel spectrometer. In principle a single channel analyzer could be used, but showness of the device would destroy the part students found most interesting. In either circumstances students might want to explore beta-ray properties, necessitating a beta spectrometer. We did not emphasize the high-resolution Ge detector. If shipping radioactive materials poses a problem, longer half-life isotopes of Pd could be used, but a dedicated radiochemical space might be needed.

It is important to note that the nuclear experiments were not repeated in subsequent terms when other faculty members taught the course. Although one of us offered to arrange for irradiations, set up the detectors and spectrometers, and review the results, the offer was not accepted. 
The number of students taking either of the theme courses remains quite small $(10-20)$. It has been postulated that inclusion of the laboratory is responsible for the small number. The students who take either course find the laboratory to be at least acceptable and often the best part of the course. We have considered dropping the lab, but doubt that the attenuated course would have nearly as much value to the students who would take it. Perhaps we should sneak it in as "demonstrations".

\section{References}

1. S. TOBIAS, Revitalizing Undergraduate Science, Research Corp, Tucson, 1992, 56-71, 174-177 [ISBN 0-9633504-1-2].

2. D. W. OXTOBY, N. H. NACHTRIEB, W. A. FREEMAN, Chemistry: Science of Change. Saunder, Philadelphia, 1990.

3. S. THOMPSON, Chemtrek: Small-Scale Experiments for General Chemistry, Allyn and Bacon, Boston, 1990 [ISBN 0-205-1 1913-1]. 